\title{
Nanoporous Gold as a Highly Selective and Active Carbon Dioxide Reduction Catalyst
}

\author{
Alex J. Welch, ${ }^{\dagger, \ddagger}$ Joseph S. DuChene, ${ }^{\dagger,}$. Giulia Tagliabue, ${ }^{\dagger, \ddagger}$ Artur Davoyan, ${ }^{\dagger+\ddagger}$ Wen-Hui Cheng, ${ }^{\dagger, \ddagger 0}$ \\ and Harry A. Atwater*, ${ }^{*}, 0$
}

${ }^{\dagger}$ Department of Applied Physics and Material Science, California Institute of Technology, Pasadena, California 91125, United States †Joint Center for Artificial Photosynthesis, California Institute of Technology, Pasadena, California 91125, United States

Supporting Information

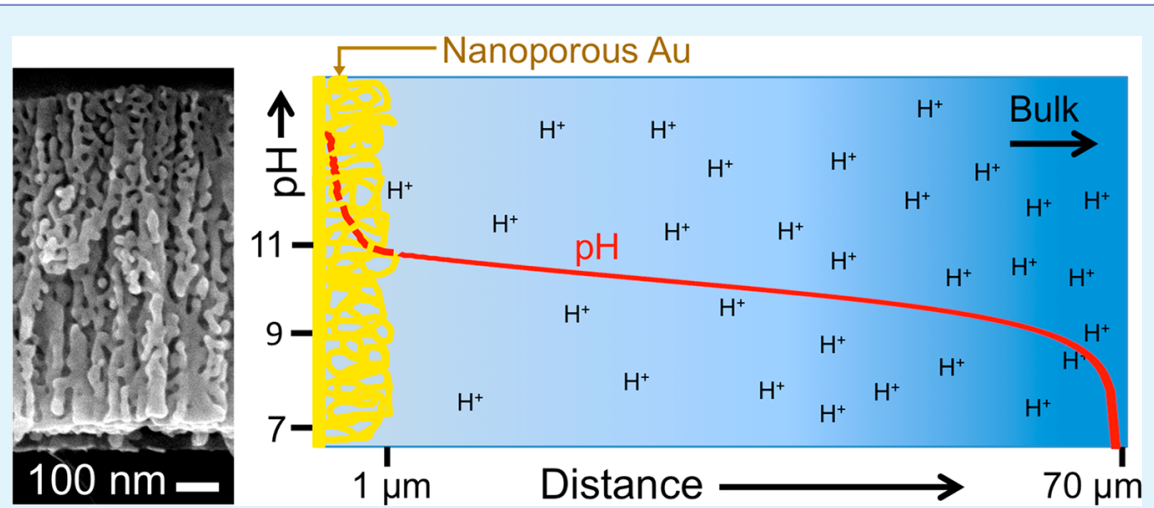

ABSTRACT: Electrochemical conversion of $\mathrm{CO}_{2}$ into useful chemicals is a promising approach for transforming $\mathrm{CO}_{2}$ into sustainably produced fuels and/or chemical feedstocks for industrial synthesis. We report that nanoporous gold (np-Au) films, with pore sizes ranging from 10 to $30 \mathrm{~nm}$, represent promising electrocatalytic architectures for the $\mathrm{CO}_{2}$ reduction reaction $\left(\mathrm{CO}_{2} \mathrm{RR}\right)$ due to their large electrochemically active surface area, relative abundance of grain boundaries, and ability to support $\mathrm{pH}$ gradients inside the nanoporous network. Electrochemical studies show that np-Au films support partial current densities for the conversion of $\mathrm{CO}_{2}$ to $\mathrm{CO}$ in excess of $6 \mathrm{~mA} \mathrm{~cm}{ }^{-2}$ at a Faradaic efficiency of $\sim 99 \%$ in aqueous electrolytes $\left(50 \mathrm{mM} \mathrm{K}_{2} \mathrm{CO}_{3}\right.$ saturated with $\mathrm{CO}_{2}$ ). Moreover, np-Au films are able to maintain Faradaic efficiency greater than $80 \%$ for CO production over prolonged periods of continuous operation $(110 \mathrm{~h})$. Electrocatalytic experiments at different electrolyte concentrations demonstrate that the pore diameter of nanoporous cathodes represents a critical parameter for creating and controlling local $\mathrm{pH}$ gradients inside the porous network of metal ligaments. These results demonstrate the merits of nanoporous metal films for the $\mathrm{CO}_{2} \mathrm{RR}$ and offer an interesting architecture for highly selective electrocatalysis capable of sustaining high catalytic currents over prolonged periods.

KEYWORDS: $\mathrm{CO}_{2}$ reduction, nanoporous cathode, $\mathrm{pH}$ gradient, grain boundaries, electrocatalysis

$\mathrm{T}$ he ability to reduce $\mathrm{CO}_{2}$ into useful chemicals or fuels will not only enable clean technology but also close the carbon cycle by recycling $\mathrm{CO}_{2}$ and preventing its further addition to the atmosphere. ${ }^{1}$ The $\mathrm{CO}_{2}$ reduction products can either be liquid fuels such as ethanol or gaseous products such as syngas $\left(\mathrm{H}_{2}\right.$ and $\left.\mathrm{CO}\right)$, which are feedstocks for thermocatalytic transformations via the Fischer-Tropsch process. $^{2-5}$ To date, $\mathrm{CO}_{2}$ reduction is not a widespread technology because of low energy efficiency associated with high overpotentials, a lack of electrocatalytic stability, and poor selectivity for the $\mathrm{CO}_{2}$ reduction reaction $\left(\mathrm{CO}_{2} \mathrm{RR}\right)$ over the $\mathrm{H}_{2}$ evolution reaction (HER), which results in low partial current densities for the product of interest. ${ }^{6}$

Various approaches have been explored to improve the activity and selectivity of Au-based electrocatalysts for the $\mathrm{CO}_{2} \mathrm{RR}$, from controlling nanocrystal size to tailoring of the exposed crystal facets, and even surface functionalization with molecular coatings. ${ }^{7-12}$ Recently, nanoporous catalytic architectures have shown promise for electrochemical $\mathrm{CO}_{2}$ reduction due to their large internal surface area and prevalence of stepped sites and grain boundaries inherent in their complex structure of highly curved metal ligaments. ${ }^{13-19}$ This propensity for under-coordinated atomic sites has been suggested to play a pivotal role in improving the selectivity of $\mathrm{CO}_{2}$ reduction in nanoporous silver (np-Ag) cathodes by stabilizing $\mathrm{CO}_{2}^{-}$intermediates involved in the electrochemical conversion of $\mathrm{CO}_{2}$ to $\mathrm{CO} .{ }^{13}$ Similar mechanisms have been invoked to explain the electrocatalytic performance of nanoporous gold (np-Au) films. ${ }^{18,19}$ While the highly irregular surface atomic structure of $\mathrm{np}-\mathrm{Au}$ is well-known, relatively less

Received: September 17, 2018

Accepted: December 26, 2018

Published: December 26, 2018 
attention has been devoted to exploring how molecular transport into and out of such a tightly confined catalytic system may also affect the selectivity for $\mathrm{CO}_{2}$ reduction within the porous network. Mesoporous Au films with controlled pore sizes around $200 \mathrm{~nm}$ in diameter have previously been shown to exhibit increased selectivity for $\mathrm{CO}_{2}$ reduction with increasing film thickness from 0.5 to $2.7 \mu \mathrm{m} .{ }^{20}$ The improved selectivity is attributed to the formation of a $\mathrm{pH}$ gradient within the porous network as protons are consumed during electrolysis faster than they can be replenished by the supporting electrolyte; this effect is increased with increasing thickness of the mesoporous metal cathode. Although this study only adjusted the overall film thickness, these observations strongly suggest that fine-tuning the metal porosity by controlling the pore size could offer a simple route to further improving the selectivity of porous cathodes for electrochemical $\mathrm{CO}_{2}$ reduction in aqueous electrolytes.

Here, we use np-Au films with pore diameters on the order of tens of nanometers to explore the influence of metal porosity on the selectivity for $\mathrm{CO}_{2}$ reduction in aqueous electrolytes. Due to their small pore diameters, the porous network of metal ligaments is able to sustain $\mathrm{pH}$ gradients within $\mathrm{np}-\mathrm{Au}$ films that are half as thick $(\sim 800 \mathrm{~nm})$ as those previously reported in mesoporous Au films $(\sim 2 \mu \mathrm{m}) .^{20}$ This effect becomes more prominent upon further decreasing the pore diameter from $\sim 30$ to $\sim 10 \mathrm{~nm}$, as evidenced by electrochemical studies. We find that np-Au films are highly selective for the conversion of $\mathrm{CO}_{2}$ to $\mathrm{CO}$ with high Faradaic efficiency $(\mathrm{FE} \sim 99 \%)$ at modest overpotentials $(\eta=0.40 \mathrm{~V})$, while at the same time delivering large partial current densities for $\mathrm{CO}\left(J_{\mathrm{CO}}=6.2 \mathrm{~mA} \mathrm{~cm}{ }^{-2}\right)$. Finally, we demonstrate that these np-Au films exhibit excellent electrochemical durability and maintain Faradaic efficiency of $\sim 80 \%$ for $\mathrm{CO}$ production over 4.5 days of continuous electrolysis at an applied potential of $E=-0.5 \mathrm{~V}$ vs the reversible hydrogen electrode (RHE).

The $\mathrm{np}-\mathrm{Au}$ films were fabricated by electron-beam deposition of $\mathrm{Ag}$ and $\mathrm{Au}$ alloys onto clean silicon $(\mathrm{Si})$ substrates, followed by selectively etching $\mathrm{Ag}$ from the $\mathrm{Ag} / \mathrm{Au}$ alloy with nitric acid (see Experimental Methods in the Supporting Information). Briefly, $2 \mathrm{~nm}$ of titanium (Ti) was initially deposited onto $\mathrm{Si}$ as an adhesion layer, followed by 50 $\mathrm{nm}$ of $\mathrm{Au}$ as a planar, nonporous base layer to support the $\mathrm{np}$ $\mathrm{Au}$ structure and to ensure that the $\mathrm{Ti}$ is not exposed to the electrolyte. The Au/Ag alloy (25/75 (vol \%)) was then codeposited in the electron beam, ranging in thickness from 100 $\mathrm{nm}$ to $2 \mu \mathrm{m}$. The np-Au morphology was then obtained by etching the $\mathrm{Au} / \mathrm{Ag}$ alloy films in $70 \mathrm{wt} \%$ nitric acid for $10 \mathrm{~min}$ at room temperature $\left(\sim 22{ }^{\circ} \mathrm{C}\right.$; denoted RT np-Au) or at low temperature $\left(-20{ }^{\circ} \mathrm{C}\right.$; denoted LT $\left.\mathrm{np}-\mathrm{Au}\right)$ in a freezer. Secondary ion mass spectrometry (SIMS) indicates that approximately 1.3 at. \% of residual Ag remains in the structure after etching, consistent with prior reports. ${ }^{21}$ Figure 1 shows helium focused ion beam (He FIB) images of np-Au samples that were etched at room temperature (Figure 1a) and at low temperature (Figure 1b), displaying average ligament thicknesses of $28 \pm 8$ and $10 \pm 2 \mathrm{~nm}$, respectively. Chemical etching at low temperatures restricts the surface mobility of the $\mathrm{Au}$ atoms during etching and ensures that the ligament diameter is decreased. ${ }^{22}$ A scanning electron microscope (SEM) cross-section image of the $\sim 800 \mathrm{~nm}$ thick RT-etched $\mathrm{np}-\mathrm{Au}$ film shows that the entirety of the film is porous down to the planar Au base layer (Figure 1c). As shown in this crosssectional image, we routinely observed that the fully etched np-

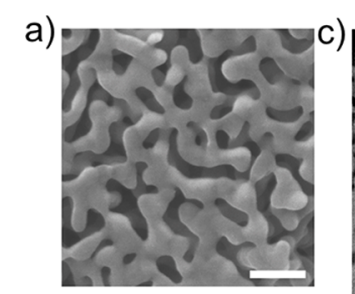

b)
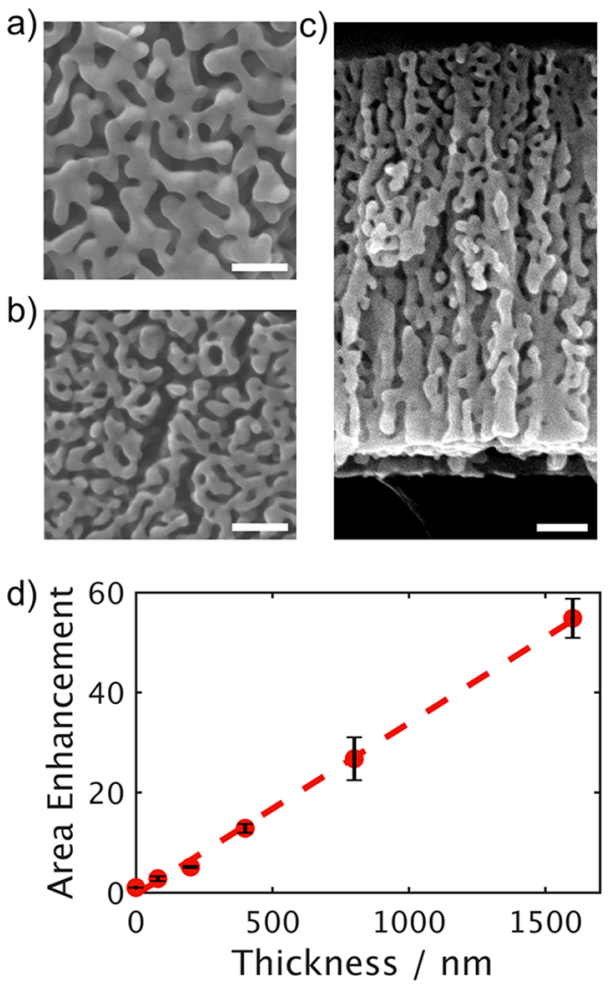

Figure 1. Helium FIB images of (a) top-down view of a nanoporous $\mathrm{Au}(\mathrm{np}-\mathrm{Au})$ film that was etched at room temperature (RT) and (b) top-down view of a np-Au film that was etched at low temperature (LT). (c) SEM cross-section image of a RT-etched np-Au film. All scale bars represent $100 \mathrm{~nm}$. (d) Electrochemical surface area enhancement as a function of film thickness for RT-etched np-Au films as determined by $\mathrm{Cu}$ underpotential deposition (UPD) experiments.

Au films were approximately $20 \%$ thinner $(\sim 800 \mathrm{~nm})$ than the initial thickness of the $\mathrm{Au} / \mathrm{Ag}$ alloy $(\sim 1 \mu \mathrm{m})$. To characterize the electrochemical surface area of the np-Au films, we performed $\mathrm{Cu}$ underpotential deposition (UPD) experiments (see Experimental Methods in the Supporting Information) and obtained a maximum roughness factor $\sim 57$ for the thickest films. It is also important to note that the surface area increases linearly with film thickness, indicating that the entire surface area of the np-Au film is electrochemically accessible (Figure 1d).

To estimate the average grain size of the np-Au films, we performed X-ray diffraction (XRD) on a RT-etched sample of $\sim 800 \mathrm{~nm}$ thickness (Supporting Information Figure S1a). For comparison, we also examined a $50 \mathrm{~nm}$ thick planar $\mathrm{Au}$ base layer and a commercial Au foil (Alfa Aesar, 99.9975\%; Figure $S 1 b, c)$. These data show that the average full width at halfmaximum of the diffraction peaks from $n p-A u$ are larger than the Au foil (Figure S1d). According to the Scherrer equation, ${ }^{23}$ the np-Au film and Au foil have average grain sizes of $20 \pm 4$ $\mathrm{nm}$ and $77 \pm 23 \mathrm{~nm}$, respectively. These calculations assume a shape factor of unity and do not take into account the possibility of microstrain. ${ }^{24}$ We also performed transmission electron microscopy (TEM) to directly visualize the distribution of grain boundaries within individual ligaments of the np-Au film. Consistent with prior reports, ${ }^{29-27}$ we observed many grain boundaries along the surface of the curved Au ligaments (Figure S2). Dark-field TEM images were also collected to estimate the average grain size within the np- 
a)

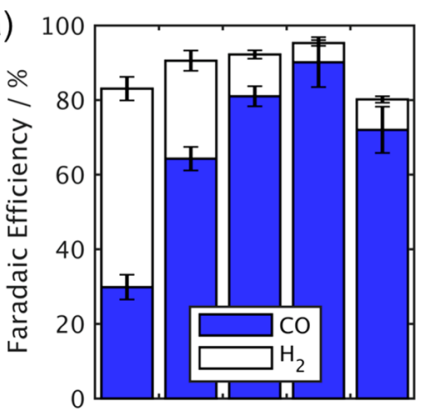

e)

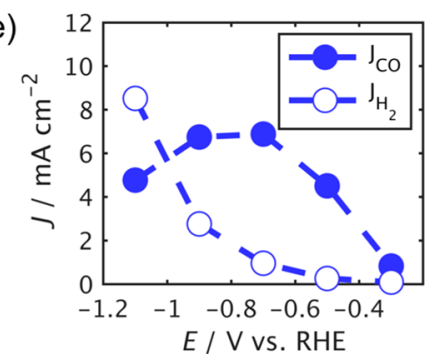

b)
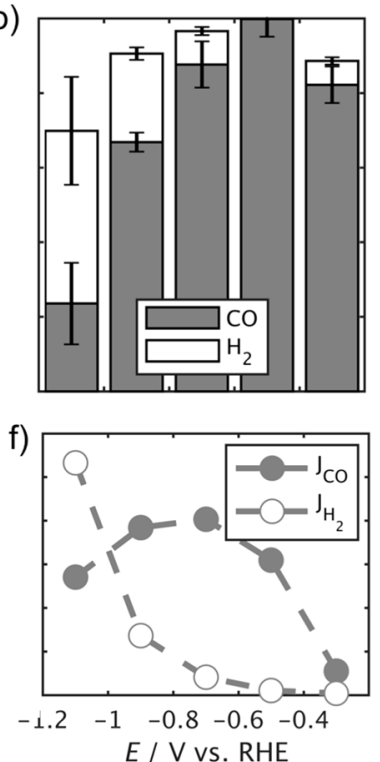
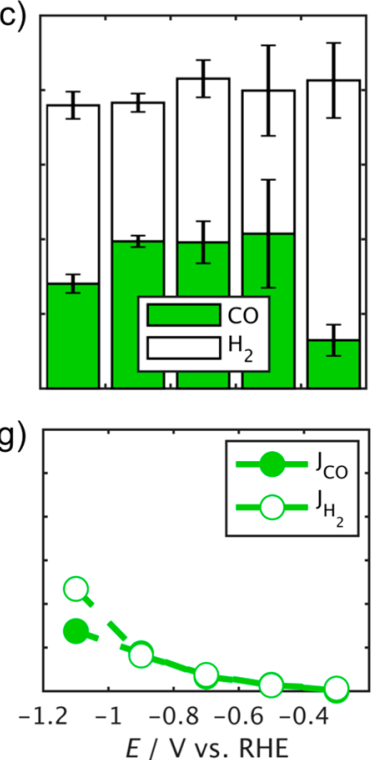
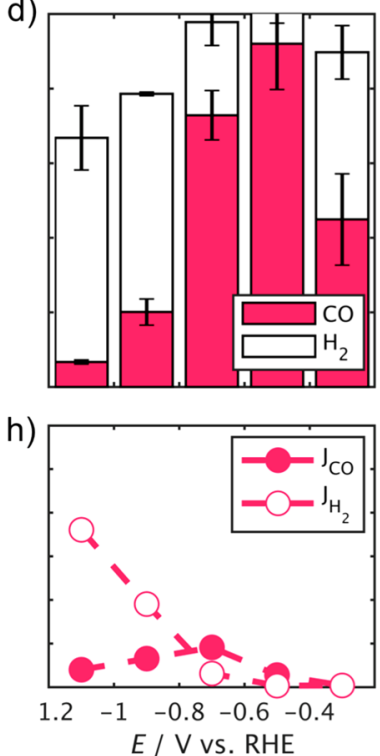

Figure 2. Electrochemical performance of Au cathodes. Faradaic efficiency (FE) for CO (filled bars) and $\mathrm{H}_{2}$ (open bars) as a function of applied potential (E) with (a) $661 \pm 10 \mathrm{~nm}$ thick RT-etched np-Au film, (b) $664 \pm 5 \mathrm{~nm}$ thick LT-etched np-Au film, (c) planar Au film, and (d) commercial Au foil. Partial current density $(J)$ for $\mathrm{CO}$ (filled circles) and $\mathrm{H}_{2}$ (open circles) as a function of applied potential for (e) $661 \pm 10$ nm thick RT np-Au film, (f) $664 \pm 5$ nm thick LT np-Au film, (g) planar Au film, and (h) commercial Au foil. Each data point represents the average FE for $\mathrm{CO}$ or $\mathrm{H}_{2}$ obtained over $2-3 \mathrm{~h}$ of continuous electrolysis at the indicated potential with $i R$ compensation. The partial current densities also represent the average value observed over the same time period. All data were obtained from the same electrode along the potential sweep.

Au film (Figure S3 and Figure S4). The average grain size that we observed in TEM $(17 \pm 4 \mathrm{~nm})$ is very similar to the estimate obtained through analysis of the XRD data. It is known that grain boundaries and under-coordinated sites in $\mathrm{Au}$ electrocatalysts serve as the active sites for $\mathrm{CO}_{2}$ reduction, ${ }^{28,29}$ suggesting that these np-Au films should exhibit substantial activity for $\mathrm{CO}_{2}$ reduction.

A two-compartment electrochemical cell was used to evaluate the electrocatalytic properties of the np-Au films for the $\mathrm{CO}_{2} \mathrm{RR}$ (see Experimental Methods in the Supporting Information). An anion exchange membrane separated the $\mathrm{Pt}$ foil anode from the np-Au cathode in $50 \mathrm{mM} \mathrm{K} \mathrm{K}_{2} \mathrm{CO}_{3}$ electrolyte saturated with dissolved $\mathrm{CO}_{2}$. It is known that higher supporting electrolyte concentrations provide higher current densities during electrolysis, ${ }^{30-32}$ but we found this concentration sufficient to enable reliable evaluation of our electrodes. The reference electrode was a leakless $\mathrm{Ag} / \mathrm{AgCl}$ electrode; all potentials are reported relative to RHE to aid comparison with literature. The cathode compartment was bubbled with $\mathrm{CO}_{2}$ at a flow rate of $5 \mathrm{~cm}^{3}(\mathrm{STP}) \mathrm{min}^{-1}$ through a glass dispersion frit to maximize the delivery of $\mathrm{CO}_{2}$ into solution during controlled potential electrolysis. The effluent gas was sent directly into a gas chromatograph (SRI instruments) to analyze the chemical products.

Figure 2a shows the FE of a RT np-Au film $(661 \pm 10 \mathrm{~nm}$ thick) for both $\mathrm{CO}$ (filled blue bars) or $\mathrm{H}_{2}$ (white bars) as a function of the applied potential $(E)$ from -0.3 to $-1.1 \mathrm{~V}_{\mathrm{RHE}}$ ( $\mathrm{V}$ vs RHE). Each data point shown in Figure 2 represents the average $\mathrm{FE}$ for $\mathrm{CO}$ or $\mathrm{H}_{2}$ obtained over $2-3 \mathrm{~h}$ of continuous electrolysis at the indicated potential with $i R$ compensation. All data were obtained from the same electrode along the potential sweep. The RT np-Au film exhibits a maximum FE for CO of $90 \%$ at $E=-0.5 \mathrm{~V}_{\mathrm{RHE}}$ with the remainder of the current producing $\mathrm{H}_{2}$. We note that no liquid products were detected for any of the Au electrodes studied. Notably, the LT np-Au film (664 $\pm 5 \mathrm{~nm}$ thick) obtains a maximum FE for CO (filled gray bars) of $99 \%$ at $E=-0.5 \mathrm{~V}_{\mathrm{RHE}}$ and maintains at least $80 \%$ FE for CO from -0.3 to $-0.7 \mathrm{~V}_{\mathrm{RHE}}$ before the HER (white bars) begins to account for a larger portion of the products at more negative applied potentials (Figure $2 \mathrm{~b}$ ). To examine the influence of the np-Au morphology on $\mathrm{CO}_{2}$ reduction selectivity, we tested the activity of a $50 \mathrm{~nm}$ thick planar $\mathrm{Au}$ film, which is the base Au layer of the np-Au electrodes. As shown in Figure $2 c$, the planar Au film primarily produces $\mathrm{H}_{2}$ (white bars) across the entire potential window; the FE for CO production (green bars) only reaches $\sim 40 \%$ at $-0.5 \mathrm{~V}_{\mathrm{RHE}}$. We also evaluated the activity of a commercial Au foil (Alfa Aeasar, 99.9975\%) to confirm that our experimental conditions and cell configuration are capable of adequately reproducing commonly observed activity trends for Au films. ${ }^{33}$ As shown in Figure 2d, the $\mathrm{Au}$ foil obtained a maximum $\mathrm{FE}$ for $\mathrm{CO}$ of $92 \%$ at $-0.5 \mathrm{~V}_{\mathrm{RHE}}$ (filled red bars), consistent with prior reports. ${ }^{33}$

A significant advantage of the np-Au morphology over the planar $\mathrm{Au}$ electrodes is illustrated by the high partial current density for $\mathrm{CO}\left(J_{\mathrm{CO}}\right)$ relative to $\mathrm{H}_{2}$ (Figure $2 \mathrm{e}-\mathrm{h}$ ). At an applied potential of $-0.7 \mathrm{~V}_{\mathrm{RHE}}$, the LT np-Au film exhibits a peak $J_{\mathrm{CO}}$ of $8.1 \mathrm{~mA} \mathrm{~cm}{ }^{-2}$ (Figure $2 \mathrm{f}$ ), which is four times higher than the Au foil (Figure $2 \mathrm{~h}$ ) and eight times higher than the planar $\mathrm{Au}$ film (Figure 2g). At the optimum applied potential for $\mathrm{CO}$ production $\left(-0.5 \mathrm{~V}_{\mathrm{RHE}}\right)$, the LT np-Au film displays $J_{\mathrm{CO}}$ of nearly $6.2 \mathrm{~mA} \mathrm{~cm}{ }^{-2}$, while the RT np-Au film $J_{\mathrm{CO}}$ is around $4.5 \mathrm{~mA} \mathrm{~cm}{ }^{-2}$. Interestingly, the LT np-Au film shows only a slight increase in $J_{\mathrm{CO}}$ as compared to that of RT np-Au despite the $\sim 3$ times increase in surface area between the LT and RT np-Au films (Figure S5a). This lower than expected $J_{\mathrm{CO}}$ from LT np-Au films likely arises due to mass transport limitations, whereby the geometry of the electrochemical cell does not allow for sufficient delivery of $\mathrm{CO}_{2}$ throughout the porous electrode to keep up with the 
a)

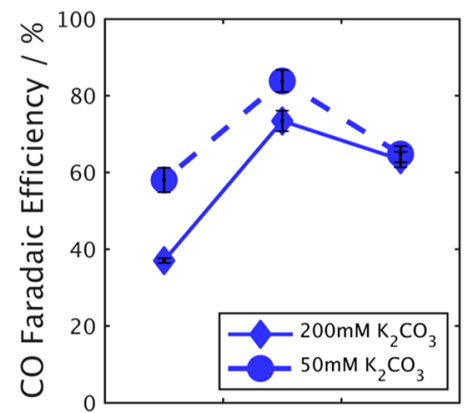

d)

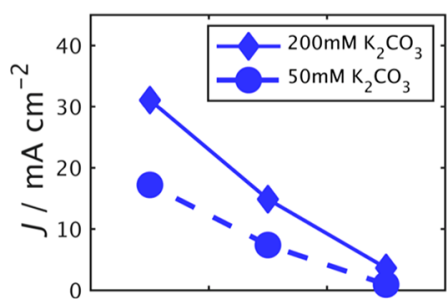

g)

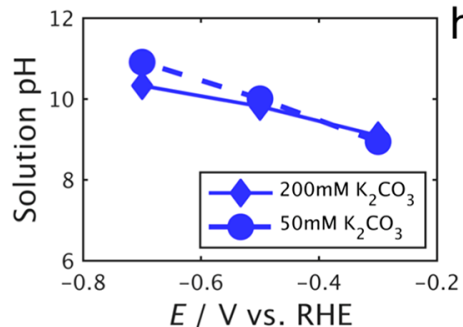

b)

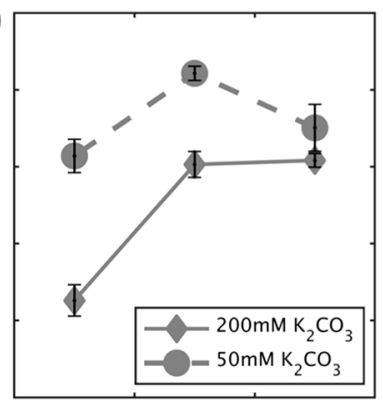

e)

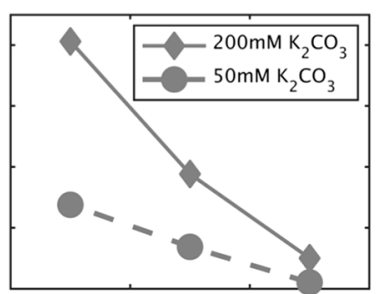

h)

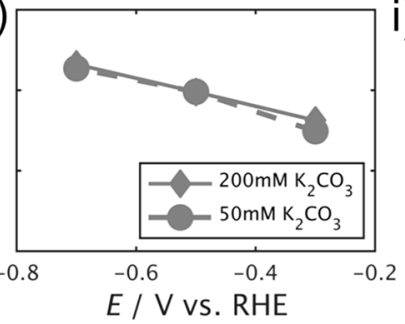

c)

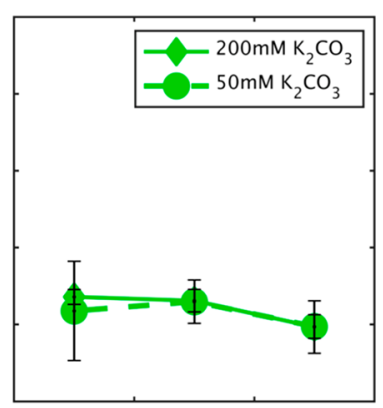

f)

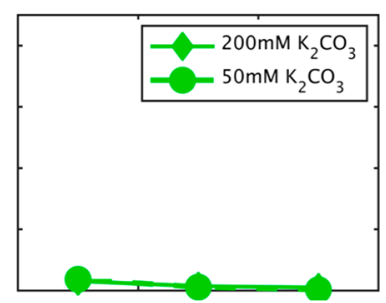

i)

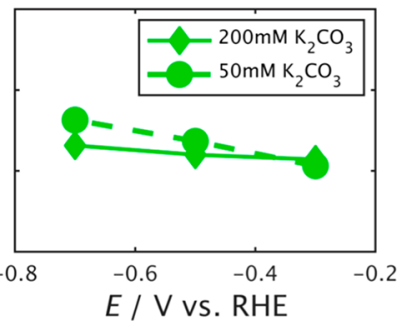

Figure 3. Influence of electrolyte concentration on $\mathrm{CO}_{2}$ reduction selectivity with Au cathodes. $(\mathrm{a}-\mathrm{c})$ Faradaic efficiency for $\mathrm{CO}$ as a function of applied potential $(E)$ obtained at two different electrolyte concentrations (both saturated with $\mathrm{CO}_{2}$ ) for (a) $809 \pm 15 \mathrm{~nm}$ thick RT-etched np-Au film, (b) $821 \pm 22 \mathrm{~nm}$ thick LT-etched np-Au film, and (c) $50 \mathrm{~nm}$ thick planar Au film. (d-f) Corresponding average current density $(J)$ obtained at $E$ observed at two different electrolyte concentrations for (d) RT np-Au, (e) LT np-Au, and (f) planar Au film. (g-i) Predicted solution pH at the surface of the electrode for (g) RT np-Au, (h) LT np-Au, and (i) planar Au film. A planar electrode geometry is assumed for the simulations.

electrochemically active surface area of the entire film. This hypothesis is confirmed by comparing the $\mathrm{FE}$ and $\mathrm{J}_{\mathrm{CO}}$ for RTetched samples that were $\sim 800$ and $\sim 150 \mathrm{~nm}$ thick (Figure S6). Despite the drastic difference in electrochemical surface area as determined by $\mathrm{Cu}$ UPD (Figure 1d), the thicker film only showed a $\sim 30 \%$ increase in $J_{\mathrm{CO}}$ under our experimental conditions (Figure S6c). Note that the linear relationship between the surface area of the np-Au film and alloy thickness (Figure 1d) implies that the entire network is accessible to the electrolyte, suggesting that a large fraction of dissolved $\mathrm{CO}_{2}$ does not penetrate the entire depth of the film at the current densities studied. These results suggest that to better use the full electrocatalytic surface area of np-Au for $\mathrm{CO}_{2}$ reduction requires that the geometry of the cell be modified to flow the $\mathrm{CO}_{2}$ directly through the np-Au film so that $\mathrm{CO}_{2}$ is efficiently delivered to the catalyst, as opposed to simply flowing the $\mathrm{CO}_{2}$ past the electrode surface. Indeed, it has recently been shown that such a tactic is highly beneficial for improving the rate of electrochemical $\mathrm{CO}_{2}$ reduction. ${ }^{34,35}$

Both the RT and LT np-Au films exhibit superior FE for CO (Figure 2a,b) relative to the planar Au film or Au foil (Figure $2 \mathrm{c}, \mathrm{d})$ across the entire potential window studied. It has previously been shown that the residual $\mathrm{Ag}$ in the np-Au film is not the source of the high FE for $\mathrm{CO} .{ }^{18}$ We therefore attribute such significant improvements in catalytic selectivity to the prevalence of grain boundaries that exist within the np-Au structure relative to the planar Au film. Another factor that likely contributes to such marked improvements in selectivity is the ability of the np-Au film to support locally alkaline $\mathrm{pH}$ conditions within the porous network as protons are consumed during electrolysis. Such an effect has previously been observed in mesoporous $\mathrm{Au}$ electrodes, which serves to suppress the rate of $\mathrm{HER}$ while the rate of $\mathrm{CO}_{2}$ reduction is relatively unaffected. $^{20}$

To examine the influence of local $\mathrm{pH}$ gradients within the nanoporous network on the selectivity of np-Au films, we examined the electrochemical activity of a RT and a LT np-Au film (Figure 3d,e) at two different electrolyte concentrations (50 mM K $\mathrm{CO}_{3}$ and $200 \mathrm{mM} \mathrm{K} \mathrm{CO}_{3}$ - both fully saturated with $\mathrm{CO}_{2}$ ). Increasing the electrolyte concentration increases the buffering capacity of the solution, ${ }^{20,32}$ which reduces any swings in local $\mathrm{pH}$ that are anticipated to form within the pores of the np-Au films as protons are consumed during electrolysis. It was therefore anticipated that the np-Au films would exhibit reduced $\mathrm{FE}$ for $\mathrm{CO}$ in $200 \mathrm{mM} \mathrm{K} \mathrm{CO}_{3}$ electrolyte if an increased solution $\mathrm{pH}$ within the porous network was responsible for the high selectivity observed on the np-Au films. As shown in Figure 3a,b, the selectivity on both RT np$\mathrm{Au}$ and LT np-Au is essentially unchanged at low applied potentials $\left(-0.3 \mathrm{~V}_{\mathrm{RHE}}\right)$, but as the current density increases with increased applied bias (Figure $3 \mathrm{~d}, \mathrm{e}$ ), any $\mathrm{pH}$ gradient that may form within the np-Au film in the $50 \mathrm{mM} \mathrm{K}_{2} \mathrm{CO}_{3}$ electrolyte (Figure 3a,b (circles)) is diminished by the improved buffering capacity of the $200 \mathrm{mM} \mathrm{K}_{2} \mathrm{CO}_{3}$ electrolyte (Figure 3a,b (diamonds)). In contrast, no change in FE for $\mathrm{CO}$ is observed on a planar Au film at any applied potential (Figure $3 \mathrm{c})$, confirming that the change in selectivity observed on the np-Au electrodes is not simply a consequence of the increased 
electrolyte concentration (Figure 3c,f). These results strongly suggest that a $\mathrm{pH}$ gradient is forming within the porous network of the Au electrocatalyst and serves an important role in achieving high selectivity of $\mathrm{CO}_{2}$ reduction in aqueous electrolytes.

Interestingly, the LT np-Au film shows a larger reduction in $\mathrm{FE}$ for $\mathrm{CO}$ (Figure $3 \mathrm{~b}$ ) than the RT np-Au film (Figure 3a). Analysis of the pore sizes between these two samples reveals that the LT np-Au film has pores with an average diameter of $10 \pm 2 \mathrm{~nm}$, while the RT np-Au sample has pores with an average diameter of $28 \pm 8 \mathrm{~nm}$ (Figure S5b). We therefore attribute the improved selectivity of the LT np-Au film relative to the RT np-Au film to the smaller pore size of the former $(\sim 10 \mathrm{~nm})$ relative to the latter $(\sim 30 \mathrm{~nm})$, which more effectively supports a high local $\mathrm{pH}$ within the porous network that improves the selectivity for $\mathrm{CO}_{2} \mathrm{RR}$ relative to HER (Figure 2a,b). Previous work has shown that increasing the thickness of a mesoporous $\mathrm{Au}$ catalyst to $\sim 2 \mu \mathrm{m}$ helps to achieve a similar effect within $\sim 200 \mathrm{~nm}$ pores. ${ }^{20}$ Our observations suggest that further reducing the pore volume below $10 \mathrm{~nm}$ may enable realizing such an effect within even thinner nanoporous metal films than those studied here.

To further explore whether the $\mathrm{pH}$ gradient is developed within the porous $\mathrm{Au}$ network or occurs largely in the boundary layer, we simulated the $\mathrm{pH}$ profile as a function of distance away from the electrode surface using the model previously reported by Gupta et al. ${ }^{30}$ Briefly, the model assumes a planar electrode geometry, which is a valid assumption for calculating the $\mathrm{pH}$ at the outer surface of the electrode because the flux of reactants and products must be the same for either a porous or planar electrocatalyst at this location. The assumption of a planar electrode is clearly incapable of accounting for changes in the transport of reactants and products into and out of the porous film itself, and we therefore interpret any experimental deviations from the model to originate from changes occurring within the porous network of metal ligaments. The inputs into the model are the electrolyte concentration, the total current density, and the Faradaic efficiency for $\mathrm{CO}$ and $\mathrm{H}_{2}$. A $70 \mu \mathrm{m}$ thick boundary layer was assumed based on the experimental flow rate of $\mathrm{CO}_{2}$ of $5 \mathrm{~cm}^{3}(\mathrm{STP}) \mathrm{min}^{-1}$ through the catholyte. ${ }^{31}$ As shown in Figure $3 \mathrm{~g}-\mathrm{i}$, these calculations predict very little change in local $\mathrm{pH}$ at the electrode surface between the two electrolyte concentrations, albeit small deviations from the bulk electrolyte are predicted for the RT np-Au film and the planar Au electrode (Figure 3g,i). While significant reductions in FE for CO were observed on both the RT and LT np-Au films (Figure 3a,b, respectively), no change in FE was observed experimentally on the planar Au film (Figure 3c). This obvious contradiction between the results of experiment with those from the model indicates that the local $\mathrm{pH}$ changes must be occurring within the porous network itself. Otherwise, we would have observed a similar reduction in FE for $\mathrm{CO}$ with the planar electrode at the higher electrolyte concentration. We note that these experimental observations are consistent with a previous report on mesoporous Au films, ${ }^{20}$ yet are achieved with much thinner films. Taken together, these results indicate that the pore diameter of porous metal electrocatalysts is a critical parameter for optimizing their selectivity and suggest that control over the pore size on the nanometer length scale may offer further improvements in electrochemical selectivity.

We further evaluated the electrocatalytic stability of these $\mathrm{Au}$ films for the $\mathrm{CO}_{2} \mathrm{RR}$ at an applied potential of $E=-0.5 \mathrm{~V}_{\mathrm{RHE}}$ (with $i R$ compensation). Significantly, we observed that the npAu film maintained a high FE for CO (nearly 90\%) over the course of $24 \mathrm{~h}$ of continuous electrocatalytic testing (Figure 4a). In stark contrast, the Au foil and planar Au films exhibit

a)

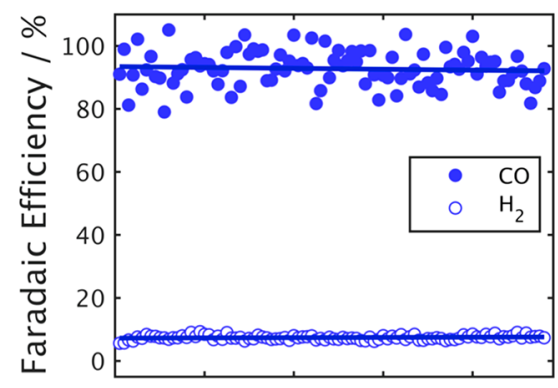

b)

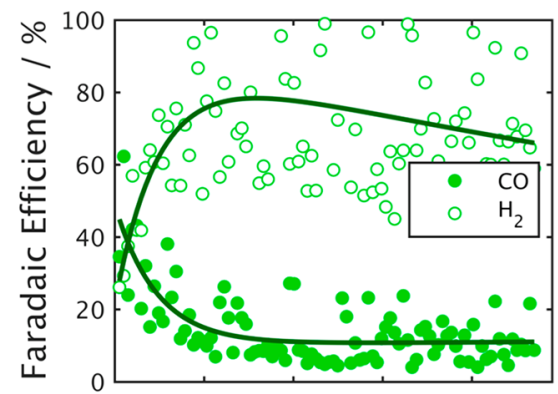

C)

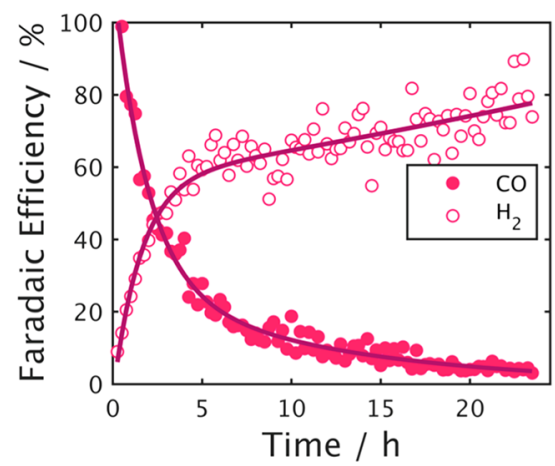

Figure 4. Extended electrochemical stability data for Au cathodes. The Faradaic efficiency for $\mathrm{CO}$ (filled circles) and $\mathrm{H}_{2}$ (open circles) was measured every $15 \mathrm{~min}$ via gas chromatography over the course of $24 \mathrm{~h}$ at an applied potential of $E=-0.5 \mathrm{~V}_{\mathrm{RHE}}$ with $i R$ compensation for (a) RT-etched np-Au, (b) planar Au film, and (c) Au foil.

drastic reductions in FE for CO over just 1 day of testing at the same applied potential (Figure $4 \mathrm{~b}, \mathrm{c}$ ). Continued testing of a different RT np-Au film for 4.5 days $(110 \mathrm{~h})$ showed continued catalytic stability (Figure S7). Comparison of the SEM images before and after testing show no significant changes in morphology except that the np-Au ligaments appear to coarsen slightly (Figure S8). Analysis of these films by XRD indicates no significant changes in peak width before and after testing, but all films showed a decrease in the overall signal magnitude from diffraction peaks associated with high-index reflections (Figure S9). We note that the activity of the Au foil can be recovered if the flame annealing treatment is repeated, but such a process is undesirable as it hinders long-term continual operation under $\mathrm{CO}_{2} \mathrm{RR}$ conditions. These observations serve to highlight the benefit of using the nanoporous metal structure to perform $\mathrm{CO}_{2}$ reduction: the prevalence of grain boundaries offers numerous active sites on the metal ligaments, while the porous network is able to support a locally alkaline 
$\mathrm{pH}$ within the film that helps improve electrocatalytic selectivity for the $\mathrm{CO}_{2} \mathrm{RR}$ over the HER.

In conclusion, we have demonstrated that $\mathrm{np}-\mathrm{Au}$ films constitute a promising electrocatalytic architecture for $\mathrm{CO}_{2}$ reduction to yield $\mathrm{CO}$ in aqueous electrolytes. The np-Au films exhibit a maximum Faradaic efficiency for CO of $99 \%$ at -0.5 $\mathrm{V}_{\mathrm{RHE}}$ while operating at a partial current density for $\mathrm{CO}$ in excess of $6 \mathrm{~mA} \mathrm{~cm}^{-2}$. We attribute the catalytic performance of $\mathrm{np}-\mathrm{Au}$ to its high electrochemical surface area possessing a large number of grain boundaries and its ability to support a local depletion of protons within the porous network. Significantly, these np-Au films maintain a Faradaic efficiency of greater than $80 \%$ over the course of $110 \mathrm{~h}$ of continuous electrolysis at $-0.5 \mathrm{~V}_{\mathrm{RHE}}$, while the activity and selectivity of both planar Au films and Au foils diminishes significantly over much shorter periods of operation $(\sim 4 \mathrm{~h})$. These studies highlight the benefits of nanoporous metal cathodes for $\mathrm{CO}_{2}$ reduction and indicate that the pore size is an important parameter to control for improving selectivity in these promising electrocatalytic architectures.

\section{ASSOCIATED CONTENT}

\section{S Supporting Information}

The Supporting Information is available free of charge on the ACS Publications website at DOI: 10.1021/acsaem.8b01570.

Materials; fabrication of nanoporous Au films; scanning electron microscopy characterization; helium focused ion beam characterization; X-ray diffraction characterization; $\mathrm{Cu}$ underpotential deposition on $\mathrm{Au}$ films; electrocatalytic reduction reaction experiments; analysis of chemical products (PDF)

\section{AUTHOR INFORMATION}

\section{Corresponding Author}

*E-mail: haa@caltech.edu;.

ORCID $\odot$

Joseph S. DuChene: 0000-0002-7145-323X

Wen-Hui Cheng: 0000-0003-3233-4606

Harry A. Atwater: 0000-0001-9435-0201

\section{Author Contributions}

A.J.W., J.S.D., G.T., and H.A.A. conceived of the experimental study. A.J.W. and J.S.D. executed all electrochemical experiments and performed the data analysis. W.-H.C. assisted with gas chromatography and high-pressure liquid chromatography. A.J.W., J.S.D., and H.A.A. wrote the paper, and all authors commented on the manuscript.

\section{Notes}

The authors declare no competing financial interest.

\section{ACKNOWLEDGMENTS}

This work is done within the Joint Center for Artificial Photosynthesis, a Department of Energy (DOE) Energy Innovation Hub, supported through the Office of Science of the U.S. Department of energy under Award Number DeSC0004993. A.J.W. acknowledges support from the National Science Foundation (NSF) Graduate Research Fellowship Program under Base Award No. 1745301. G.T. acknowledges support from the Swiss National Science Foundation through the Advanced Mobility Fellowship, grant n. P300P2_171417. We gratefully acknowledge critical support and infrastructure provided for this work by the Kavli Nanoscience Institute at
Caltech. We thank Matthew S. Hunt of the Kavli Nanoscience Institute at Caltech for assistance with SEM, He FIB, and TEM imaging of nanoporous Au films. Any opinions, findings, and conclusions expressed in this material are those of the authors and do not necessary reflect those of DOE or NSF.

\section{REFERENCES}

(1) Jones, J.-P.; Prakash, G. K. S.; Olah, G. A. Electrochemical $\mathrm{CO}_{2}$ Reduction: Recent Advances and Current Trends. Isr. J. Chem. 2014, 54, 1451-1466.

(2) White, J. L.; Baruch, M. F.; Pander, J. E.; Hu, Y.; Fortmeyer, I. C.; Park, J. E.; Zhang, T.; Liao, K.; Gu, J.; Yan, Y.; Shaw, T. W.; Abelev, E.; Bocarsly, A. B. Light-Driven Heterogeneous Reduction of Carbon Dioxide: Photocatalysts and Photoelectrodes. Chem. Rev. 2015, 115, 12888-12935.

(3) Whipple, D.; Kenis, P. Prospects of $\mathrm{CO}_{2}$ Utilization via Direct Heterogeneous Electrochemical Reduction. J. Phys. Chem. Lett. 2010, $1,3451-3458$.

(4) Kuhl, K. P.; Hatsukade, T.; Cave, E. R.; Abram, D. N.; Kibsgaard, J.; Jaramillo, T. F. Electrocatalytic Conversion of Carbon Dioxide to Methane and Methanol on Transition Metal Surfaces. J. Am. Chem. Soc. 2014, 136, 14107-14113.

(5) Kuhl, K. P.; Cave, E. R.; Abram, D. N.; Jaramillo, T. F. New Insights into the Electrochemical Reduction of Carbon Dioxide on Metallic Copper Surfaces. Energy Environ. Sci. 2012, 5, 7050-7059.

(6) Hori, Y. Electrochemical $\mathrm{CO}_{2}$ Reduction on Metal Electrodes. In Modern Aspects of Electrochemistry; Vayenas, C. G., White, R. E., Gamboa-Aldeco, M. E., Eds.; Springer: New York, NY, USA, 2008; pp 89-189.

(7) Cao, Z.; Kim, D.; Hong, D.; Yu, Y.; Xu, J.; Lin, S.; Wen, X.; Nichols, E. M.; Jeong, K.; Reimer, J. A.; Yang, P.; Chang, C. J. A Molecular Surface Functionalization Approach to Tuning Nanoparticle Electrocatalysts for Carbon Dioxide Reduction. J. Am. Chem. Soc. 2016, 138, 8120-8125.

(8) Fang, Y.; Flake, J. C. Electrochemical Reduction of $\mathrm{CO}_{2}$ at Functionalized Au Electrodes. J. Am. Chem. Soc. 2017, 139, 33993405.

(9) Zhu, W.; Michalsky, R.; Metin, Ö.; Lv, H.; Guo, S.; Wright, C. J.; Sun, X.; Peterson, A. A.; Sun, S. Monodisperse Au Nanoparticles for Selective Electrocatalytic Reduction of $\mathrm{CO}_{2}$ to CO. J. Am. Chem. Soc. 2013, 135, 16833-16836.

(10) Zhu, W.; Zhang, Y.-J.; Zhang, H.; Lv, H.; Li, Q.; Michalsky, R.; Peterson, A. A.; Sun, S. Active and Selective Conversion of $\mathrm{CO}_{2}$ to CO on Ultrathin Au Nanowires. J. Am. Chem. Soc. 2014, 136, 1613216135 .

(11) Chen, Y.; Li, C. W.; Kanan, M. W. Aqueous $\mathrm{CO}_{2}$ Reduction at Very Low Overpotential on Oxide-Derived Au Nanoparticles. J. Am. Chem. Soc. 2012, 134, 19969-19972.

(12) Liu, M.; Pang, Y.; Zhang, B.; De Luna, P.; Voznyy, O.; Xu, J.; Zheng, X.; Dinh, C. T.; Fan, F.; Cao, C.; de Arquer, F. P. G.; Safaei, T. S.; Mepham, A.; Klinkova, A.; Kumacheva, E.; Filleter, T.; Sinton, D.; Kelley, S. O.; Sargent, E. H. Enhanced Electrocatalytic $\mathrm{CO}_{2}$ Reduction via Field-Induced Reagent Concentration. Nature 2016, 537, 382.

(13) Lu, Q.; Rosen, J.; Zhou, Y.; Hutchings, G. S.; Kimmel, Y. C.; Chen, J. G.; Jiao, F. A Selective and Efficient Electrocatalyst for Carbon Dioxide Reduction. Nat. Commun. 2014, 5, 3242.

(14) Dutta, A.; Morstein, C. E.; Rahaman, M.; Cedeño López, A.; Broekmann, P. Beyond Copper in $\mathrm{CO}_{2}$ Electrolysis: Effective Hydrocarbon Production on Silver-Nanofoam Catalysts. ACS Catal. 2018, 8, 8357-8368.

(15) Sen, S.; Liu, D.; Palmore, G. T. R. Electrochemical Reduction of $\mathrm{CO}_{2}$ at Copper Nanofoams. ACS Catal. 2014, 4, 3091-3095.

(16) Ma, M.; Trześniewski, B. J.; Xie, J.; Smith, W. A. Selective and Efficient Reduction of Carbon Dioxide to Carbon Monoxide on Oxide-Derived Nanostructured Silver Electrocatalysts. Angew. Chem., Int. Ed. 2016, 55, 9748-9752. 
(17) Zhang, Y.; Luc, W.; Hutchings, G. S.; Jiao, F. Photoelectrochemical Carbon Dioxide Reduction Using a Nanoporous Ag Cathode. ACS Appl. Mater. Interfaces 2016, 8, 24652-24658.

(18) Zhang, W.; He, J.; Liu, S.; Niu, W.; Liu, P.; Zhao, Y.; Pang, F.; Xi, W.; Chen, M.; Zhang, W.; et al. Atomic Origins of High Electrochemical $\mathrm{CO}_{2}$ Reduction Efficiency on Nanoporous Gold. Nanoscale 2018, 10, 8372-8376.

(19) Chen, C.; Zhang, B.; Zhong, J.; Cheng, Z. Selective Electrochemical $\mathrm{CO}_{2}$ Reduction over Highly Porous Gold Films. J. Mater. Chem. A 2017, 5, 21955-21964.

(20) Hall, A. S.; Yoon, Y.; Wuttig, A.; Surendranath, Y. Mesostructure-Induced Selectivity in $\mathrm{CO}_{2}$ Reduction Catalysis. J. Am. Chem. Soc. 2015, 137, 14834-14837.

(21) Biener, J.; Biener, M. M.; Madix, R. J.; Friend, C. M. Nanoporous Gold: Understanding the Origin of the Reactivity of a 21 st Century Catalyst Made by Pre-Columbian Technology. ACS Catal. 2015, 5, 6263-6270.

(22) Qian, L. H.; Chen, M. W. Ultrafine Nanoporous Gold by LowTemperature Dealloying and Kinetics of Nanopore Formation. Appl. Phys. Lett. 2007, 91, 083105.

(23) Patterson, A. L. The Scherrer Formula for X-Ray Particle Size Determination. Phys. Rev. 1939, 56, 978-982.

(24) Jiang, H. G.; Rühle, M.; Lavernia, E. J. On the Applicability of the X-Ray Diffraction Line Profile Analysis in Extracting Grain Size and Microstrain in Nanocrystalline Materials. J. Mater. Res. 1999, 14, 549-559.

(25) Hodge, A. M.; Biener, J.; Hsiung, L. L.; Wang, Y. M.; Hamza, A. V.; Satcher, J. H. Monolithic Nanocrystalline Au Fabricated by the Compaction of Nanoscale Foam. J. Mater. Res. 2005, 20, 554-557.

(26) Mathur, A.; Erlebacher, J. Size Dependence of Effective Young's Modulus of Nanoporous Gold. Appl. Phys. Lett. 2007, 90, 061910.

(27) Petegem, S. V.; Brandstetter, S.; Maass, R.; Hodge, A. M.; ElDasher, B. S.; Biener, J.; Schmitt, B.; Borca, C.; Swygenhoven, H. V. On the Microstructure of Nanoporous Gold: An X-Ray Diffraction Study. Nano Lett. 2009, 9, 1158-1163.

(28) Mariano, R. G.; McKelvey, K.; White, H. S.; Kanan, M. W. Selective Increase in $\mathrm{CO}_{2}$ Electroreduction Activity at GrainBoundary Surface Terminations. Science 2017, 358, 1187.

(29) Feng, X.; Jiang, K.; Fan, S.; Kanan, M. W. Grain-BoundaryDependent $\mathrm{CO}_{2}$ Electroreduction Activity. J. Am. Chem. Soc. 2015, 137, 4606-4609.

(30) Gupta, N.; Gattrell, M.; MacDougall, B. Calculation for the Cathode Surface Concentrations in the Electrochemical Reduction of $\mathrm{CO}_{2}$ in $\mathrm{KHCO}_{3}$ Solutions. J. Appl. Electrochem. 2006, 36, 161-172.

(31) Clark, E. L.; Resasco, J.; Landers, A.; Lin, J.; Chung, L.-T.; Walton, A.; Hahn, C.; Jaramillo, T. F.; Bell, A. T. Standards and Protocols for Data Acquisition and Reporting for Studies of the Electrochemical Reduction of Carbon Dioxide. ACS Catal. 2018, 8, 6560-6570.

(32) Singh, M. R.; Clark, E. L.; Bell, A. T. Effects of Electrolyte, Catalyst, and Membrane Composition and Operating Conditions on the Performance of Solar-Driven Electrochemical Reduction of Carbon Dioxide. Phys. Chem. Chem. Phys. 2015, 17, 18924-18936.

(33) Cave, E. R.; Montoya, J. H.; Kuhl, K. P.; Abram, D. N.; Hatsukade, T.; Shi, C.; Hahn, C.; Nørskov, J. K.; Jaramillo, T. F. Electrochemical $\mathrm{CO}_{2}$ Reduction on Au Surfaces: Mechanistic Aspects Regarding the Formation of Major and Minor Products. Phys. Chem. Chem. Phys. 2017, 19, 15856-15863.

(34) Dinh, C. T.; Burdyny, T.; Kibria, M. G.; Seifitokaldani, A.; Gabardo, C. M.; García de Arquer, F. P.; Kiani, A.; Edwards, J. P.; De Luna, P.; Bushuyev, O. S.; Zou, C.; Quintero-Bermudez, R.; Pang, Y.; Sinton, D.; Sargent, E. H. $\mathrm{CO}_{2}$ Electroreduction to Ethylene via Hydroxide-Mediated Copper Catalysis at an Abrupt Interface. Science 2018, 360, 783.

(35) Hoang, T. T. H.; Ma, S.; Gold, J. I.; Kenis, P. J. A.; Gewirth, A. A. Nanoporous Copper Films by Additive-Controlled Electrodeposition: $\mathrm{CO}_{2}$ Reduction Catalysis. ACS Catal. 2017, 7, 33133321. 